for a year, and it was found that the right eye was blind, while the left showed temporal hemianopsia. Thyroid extract caused great improvement in the general condition and in the visual symptoms in particular, for central vision rose and the temporal part of the field was partly regained. Unfortunately the state of the right eye was too far gone for much improvement to take place.-Arch. d'oph., Paris, 1907, September.

\title{
An Unusual Cause of Recurrent Conjunctivitis.
}

Every ophthalmic surgeon recognises cases of recurrent conjunctivitis, returning without any apparent reason whatever, the mucous membrane being sometimes free from all bacterial growth. Risley mentions two cases in which the cause of this condition seemed fairly clearly proved, though it is a very unusual one. The first was that of a lady, æt. 25, who cannot come into the neighbourhood of a horse without an access of what one might call asthma, analogous to "hay fever." The nostrils becomes tuffed, there is persistent sneezing and profuse lachrymation. The eyes will remain red and painful for hours after even only a few minutes' proximity to a horse. Naturally this lady is an enthusiastic upholder of the automobile! The other case is also that of a young lady who presents exactly similar symptoms when in the immediate neighbourhood of a cat. Of course cases of cat-asthma are well known, but the reviewer has never traced conjunctivitis either to horse or cat. Is it possible that some other cases may have their origin in such "poisonous" plants as the Primula obconica?-Trans. Am. Ophth. Soc., Hertford, Conn., 1907, vol. xi.

\section{REPORTS OF THE MEDICAL SOCIETIES.}

Edinburgh Medico-Chirurgiçal Society.-May 6, 1908.-Dr. Jayes Ritchie, President, in the chair.-Dr. T. D. Luke showed-(1) a new dropper for ethyl chloride; (2) an open ether mask.-Professor ChiEne described-(1) A simple method of preparing catgut; (2) how to demonstrate colour-photographic transparancies without a lantern.Dr. H. Melville Dunlop read a paper entitled "Some Considerations regarding Pneumonia in Children, drawn from an Analysis of 500 Cases," which will appear in the Journal.

A clinical meeting, illustrative of the subject of Dermatology, was held in the Royal Infirmary on May 20, 1908-Dr. James Ritchie, President, in the chair.-Dr. Norman Walker gave a résumé of the work of the Skin Department of the Infirmary during the past sixteen months, with special reference to interesting groups of cases, and to those subjects at present attracting attention. He first referred to alopecia areata. This disease appeared to be about twice as frequent, relatively to the total number of cases seen, as in either London or Glasgow. This, he thought, supported the view that the disease is infectious. Favus 
was next referred to ; it was notoriously common in Edinburgh, but he doubted whether many of his audience realised that he could have collected, as he wished, sixty cases to show. The disease in private practice can be cured readily enough ; among the poor, however, it was frequently incurable. The reason for this was simply that the patients would not attend regularly. Favus could easily be stamped out if means could be devised to compel the children to attend hospital regularly. Impetigo contagiosa showed a definite epidemicity. In some months (not the same in each year) the disease was much more common than in others. Herpes zoster also showed a similar incidence. Lupus erythematosus was held by some authorities, particularly in France, to be tuberculous in origin. It was not held that the lesion was due to a local tuberculosis, but that it was caused by the toxins of tubercle bacillus located in a distant focus. Dr. Walker had tried von Pirquet's cutaneous reaction in several cases, but he only got one positive reaction. This, he thought, was strongly against the theory that the disease is tuberculous. He protested against the indiscriminate use of Calmette's reaction, as a patient on whom he tried it nearly lost the sight of one eye. Speaking of lupus vulgaris, he said that in using the X-rays he had learned the importance of patient, prolonged, and persistent treatment. He showed several cases which were practically cured after years of treatment. He regarded the use of oleate of mercury as occupying nearly as high a place in the therapeutics of lupus as the $\mathrm{X}$-rays themselves. He had been struck by the number of times he had seen epithelioma develop in the course of the X-ray treatment of this disease. Several cases were shown to illustrate this. The appearance of cancer did not mean that the rays must necessarily be discontinued. It certainly was wrong, in his opinion, to say that if epithelioma developed larger doses of the rays were indicated. Molluscum contagiosum was not a very common disease in his series. He had noted one group of five cases of this disease in which all the patients had frequented the same public baths. Acne rosaceæ was commonly ascribed to digestive disorder; he was able to say that this was relatively rare in his cases. He thought there was a connection between this disease and seborrhœa. Regarding ringworm, he had not had the same success with $\mathrm{X}$-rays in this disease as was obtained in Paris. Referring to the general question of vaccine therapy, Dr. Walker showed a severe case of sycosis which had been rapidly cured with vaccinations of his own staphylococci. In lupus, if tuberculin was to be of any use, it must be given in some way which did not necessitate the estimation of the opsonic index. His practice was to give $\frac{1}{1500} \mathrm{mgrm}$. of tuberculin every four weeks, and he was satisfied with it as an adjuvant to local treatment. Dr. GARDNER showed-(1) A somewhat rare form of erythema multiforme; (2) a patient with recurrence of ringworm of the scalp. In the first attack the small spore fungus, in this the large spore fungus, had been found.-Dr. Cranston Low showed-(1) A patient who had previously suffered from an attack of dermatitis herpetiformis, and now had an attack of pemphigus foliaceus; (2) a case of commencing mycosis fungoides; (3). [for Mr. Miles] a patient with unusually wellmarked rupia.-Dr. J. S. FowLER showed an infant suffering from urticaria pigmentosa.-Mr. HENRY WADE showed specimens of- 
(1) Hyperkeratosis plantaris ; (2) mycetoma.-A demonstration of casts, photographs, etc., was also given in connection with the meeting.

Edinburgh Obstetrical Societr.-May 13, 1908.-Professor Stephenson, President, in the chair.-Dr. Munno Kerr, Glasgow, read notes on a "Case of Angular Pregnancy" which very closely resembled an ectopic gestation. Pain and hæmorrhage were complained of for some days, and a small tumour was discovered in the neighbourhood of the right tube. Two days later a decidual cast was shed, but no ovum. The ovum with remains of the cast was expelled a day or two after, and the swelling in the neighbourhood of the tube disappeared. He also described a case in which death of a fœtus in utero occurred at the thirty-sixth week. The fœtal heart sounds underwent a distinct change for a week previous to death. He also gave a communication on rupture of the uterus, with remarks on diagnosis and treatment, based on a series of fourteen cases. In most of his cases the pulse-rate had never been above 90 , the temperature frequently subnormal, and the hæmorrhage not great in amount. He considered that plugging was the best treatment to be adopted in cases of rupture, and he preferred pan-hysterectomy to sub-total hysterectomy in cases where operative interference was required.-Dr. W. Fordyce gave an account of an interesting case of a patient, æt. 48, on whom he had performed hysterectomy for fibroid tumour under spinal anæsthesia. On two previous occasions operation had been deemed inadvisable on account of prevailing cardiac symptoms, but, owing to the severity of the pain and profuse hæmorrhage, along with the expressed desire of the patient, operation was ultimately performed. About 15 c.c. of cerebrospinal fluid were withdrawn, and 6 cgrms. of tropacocaine diluted with 5 c.c. of the cerebro-spinal fluid were injected into the subarachnoid space between the third and fourth lumbar spines by Mr. J. W. Struthers. The effect of the anæsthesia lasted for forty-five minutes, during which time the operation was successfully carried out, and the patient made an uninterrupted recovery. There were slight murmurs in the mitral and aortic regions, but Dr. Fordyce was of the opinion that the cardiac symptoms were more due to a general malnutrition than to any organic valvular lesion.

Glasgow Medico-Chirurgical Society.-Friday, May 1, 1908.Dr. Walker Downie, President, in the chair.-Dr. James Adam read a paper on "The Pathology and Treatment of Atrophic Rhinitis." He also showed a series of patients and microscopic specimens illustrating the paper. The condition was usually associated with sinus disease and Abel's bacillus was frequently met with.-Mr. J. H. Pringle showed a patient after inter-ilio-abdominal amputation. The leg had been amputated for sarcoma, and recurrence took place in the ischium. The half of the pelvis (from the sacro-iliac synchondrosis almost to the symphysis) was removed. The patient did well, and there was no hernia afterwards. He got about, using a crutch on the side of the sound limb.-Drs. T. K. Monro and Leonard Findlay showed preparations from a case of general tuberculosis.-Dr. T. K. Monro also read notes on a "Case of Hyperpyrexia in Rheumatism successfully treated by the Cold Bath." 